\title{
The relationship between perceived length and egocentric location in Müller-Lyer figures with one versus two chevrons
}

\author{
ROBERT B. WELCH \\ NASA Ames Research Center, Moffett Field, California \\ ROBERT B. POST \\ University of California, Davis, California \\ WAYLAND LUM \\ California State University, San Jose, California \\ and \\ WILLIAM PRINZMETAL \\ University of California, Berkeley, California
}

\begin{abstract}
We examined the apparent dissociation of perceived length and perceived position with respect to the Müller-Lyer (M-L) illusion. With the traditional (two-chevron) figure, participants made accurate open-loop pointing responses at the endpoints of the shaft, despite the presence of a strong length illusion. This apparently non-Euclidean outcome replicated that of Mack, Heuer, Villardi, and Chambers (1985) and Gillam and Chambers (1985) and contradicts any theory of the M-L illusion in which mislocalization of shaft endpoints plays a role. However, when one of the chevrons was removed, a constant pointing error occurred in the predicted direction, as well as a strong length illusion. Thus, with onechevron stimuli, perceived length and location were no longer completely dissociated. We speculated that the presence of two opposing chevrons suppresses the mislocalizing effects of a single chevron, especially for figures with relatively short shafts.
\end{abstract}

Roger Shepard claimed that "Throughout biological evolution, the most pervasive and enduring constraints governing the external world ... must have become most deeply incorporated into our perceptual machinery. Especially basic are ... that space is locally Euclidean" (Shepard, 1981, p. 279, italics added). However, there is evidence to suggest that perceptual and Euclidean spaces are not always congruent. For example, with "induced motion," errors in pointing at the apparently moving target are substantially smaller than would be predicted by the amount of reported apparent motion (e.g., Abrams \& Landgraf, 1990). Another example, and the subject of the present article, is the Müller-Lyer (M-L) illusion. This well-known configuration consists of a shaft with an opposing chevron at each end. When the tails of these chevrons point outward (Figure 1A), the shaft appears longer than when they point inward (Figure 1B). If perceptual space always obeys the rules of Euclidean geometry, this illusion should also entail

This work was supported by the National Aeronautics and Space Administration (Project UPN 199-16-12-34). We thank Arnold Stoper for his comments on an earlier draft of this manuscript. Correspondence should be addressed to R. B. Welch, NASA Ames Research Center, Mail Stop 262-2, Moffett Field, CA 94035 (e-mail: robert.b.welch@nasa.gov).

Note-This article was accepted by the previous editorial team, headed by Neil Macmillan. errors in egocentric location. That is, the shaft endpoints of Figure 1A should appear displaced outward from their true loci by an amount comparable to the shaft overestimation, and the shaft endpoints of Figure 1B should appear displaced inward. However, studies by Mack et al. (1985) and Gillam and Chambers (1985), described below, suggest otherwise. The present investigation expanded upon these results by examining the role of the two opposing chevrons of the M-L configuration in the apparent dissociation between perceived length and egocentric location.

The most common way of assessing perceived M-L shaft length is to have observers attempt to equate the shaft length of one of the critical figures to that of the other critical figure (or to a control figure such as Figure 1C). Egocentric location of the endpoints, on the other hand, has been measured in several qualitatively different ways. Gillam and Chambers (1985) had their participants indicate when a dot, located $60 \mathrm{~cm}$ beneath the M-L figure, appeared to be directly below one or the other of the endpoints. The investigators found no illusion of location. However, Morgan, Hole, and Glennerster (1990) have argued that this task might be a measure of the perceived orientation between the two targets, rather than endpoint location. A second potential measure of egocentric localization is saccadic eye movement. For example, if observers who are attempting to saccade be- 
A

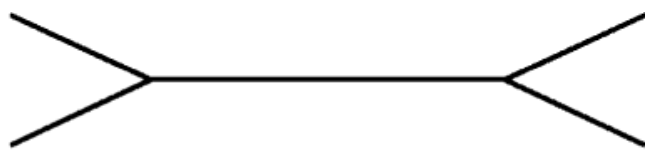

B
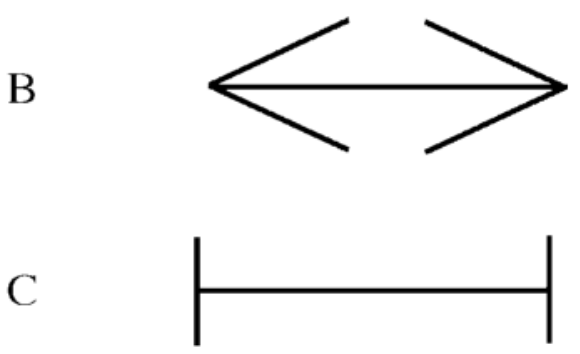

Figure 1. (A) Tails-out, (B) tails-in, and (C) tails-up (control) Müller-Lyer figures.

tween the two M-L shaft endpoints were to overshoot the target in the presence of the tails-out figure and undershoot it with the tails-in figure, one might be tempted to conclude that the illusion involves egocentric mislocalization. However, it is now known that saccadic eye movements are not influenced by perceived location (e.g., Wong \& Mack, 1981), and thus, even if the M-L illusion does entail mislocalization, this procedure could not detect it. A third way of measuring egocentric localization is by means of open-loop pointing or reaching. Thus, with this procedure (e.g., Mack et al., 1985), participants are instructed to place the unseen index finger at a given M-L shaft endpoint. If the M-L length illusion is accompanied by comparable errors in egocentric localization, open-loop pointing should err in a manner compatible with the illusion. Because this measure is clearly one of egocentric location and does not seem susceptible to the criticisms of the procedures described above, we chose it as the method for the present investigation.

On the basis of open-loop pointing, Mack et al. (1985) found no egocentric localization errors in their Experiment 1, but small, statistically reliable errors in Experiments 2 and 3 . A possible explanation for the results of the latter experiments is that, contrary to the first experiment, observers were not allowed to point at the figure until after it was out of view. This procedure may introduce a memory component likely to favor the use of perceived length, rather than perceived location, to guide pointing. Gentilucci, Chieffi, Daprati, Saetti, and Toni (1996) reported a similar finding. Mack et al. argued that the very large differences between length and egocentric localization measures found in all three of their experiments indicated that these perceptual capacities are essentially dissociated from one another with respect to the M-L illusion.

The goal of the first of the present experiments was to confirm the results of Experiment 1 of Mack et al. (1985). The remaining experiments tested the hypothesis that the source of the dissociation between perceived length and location in the M-L illusion is the presence of the two opposing chevrons. It was predicted that by removing one of the chevrons, the two perceptions would become partially or perhaps completely reassociated; that is, that the illusion of length would now be accompanied by an illusion of location.

\section{EXPERIMENT 1}

The first experiment was a replication of Experiment 1 of Mack et al. (1985). On the basis of the previous arguments, we decided that visual egocentric localization should be measured by means of open-loop pointing at currently viewed targets. Because Mack et al. fulfilled these criteria only in their first experiment, the remaining comparisons between their research and ours will refer to that experiment.

\section{Method}

Participants. The initial sample of 12 participants consisted of both introductory psychology students from a local university taking part in the experiment to fulfill a course requirement and individuals (both students and nonstudents) who were paid for their services. The only selection restriction was the possession of 20-20 visual acuity (normally or with correction). Unfortunately, some of the participants failed to evidence an illusion with respect to the tails-in figure, a not uncommon experience in M-L illusion studies. Because the primary goal of this experiment was to determine whether a measurable M-L illusion of extent is accompanied by errors in egocentric localization, these participants were replaced. On the basis of this a priori criterion, 3 participants were substituted. The final sample consisted of 8 males and 4 females, with an average age of approximately 25 years.

Procedure. For each of the critical M-L figures (tails-in and tailsout), and the control figure (tails-up), observers were measured on (1) visually perceived shaft length and (2) accuracy in open-loop pointing at the two shaft endpoints. The order of the visual and pointing trial blocks was counterbalanced across participants, as was the order in which the three M-L figures were presented.

On each trial, the participant placed his/her face firmly against a pair of goggles and looked directly at one of the three horizontally oriented M-L figures drawn on a sheet of white paper affixed to a vertical foam-core surface. On length measurement trials, the participants viewed a given figure for 1-2 sec and then looked down at the table at an apparatus containing a horizontal line whose length could be adjusted by means of a sliding panel. Using the visible right hand (even if left-handed), the participants moved the panel in or out (according to an in/out/out/in sequence). The task was to adjust the horizontal line until it appeared to be the same length as the figure just viewed. The instructions made it very clear that the portion of the M-L figure to be matched was the horizontal segment connecting the vertices of the two chevrons (i.e., the shaft) and thus was not to include the chevrons themselves. ${ }^{1}$ The experimenter, who sat directly opposite the participant, read and recorded the setting of the adjustable line from a ruler located on his side of the apparatus. The apparatus shielded the participant's view of both the experimenter and the ruler. A block of eight trials was performed for each of the three M-L figures.

For the egocentric location measurements, participants peered through the goggles at a given M-L figure and reached beneath a horizontal occluding board with the unseen right index finger (even if left-handed), pointing first at one shaft endpoint and then the other for a total of four trials for each endpoint. Pointing accuracy was measured by comparing the location of the finger with a metric ruler on the experimenter's side of the apparatus.

Stimuli. The figures were located approximately $20.7 \mathrm{~cm}$ from the lenses of the participant's eyes at approximately eye level. The 


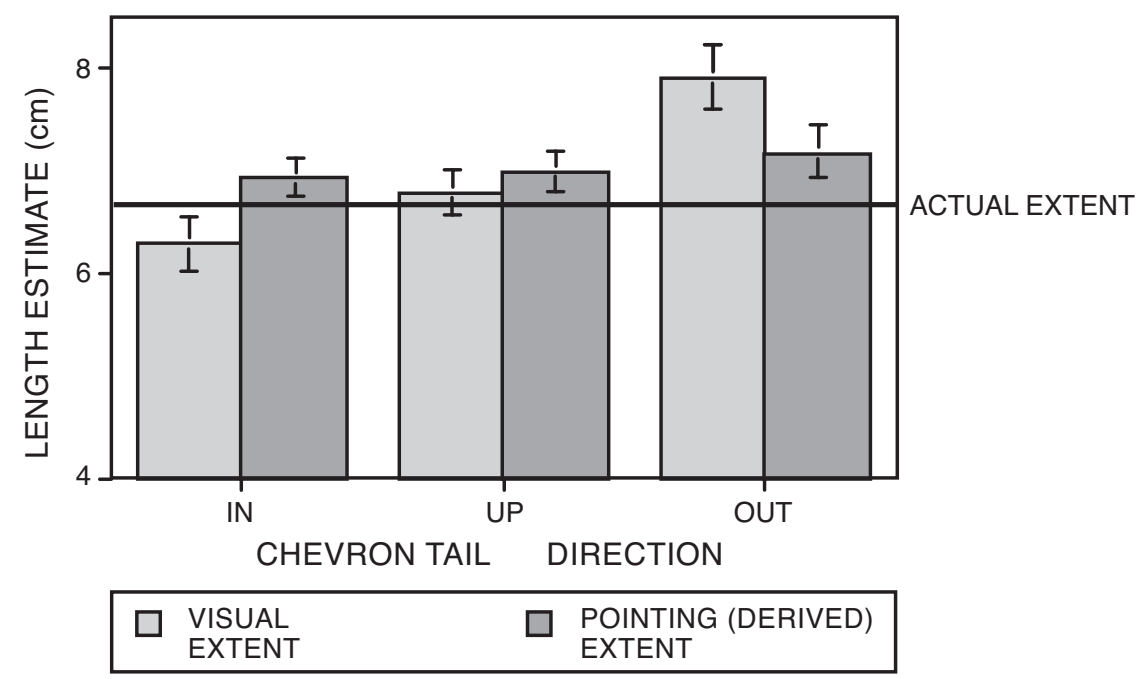

Figure 2. Experiment 1: Visual and pointing (derived) estimates of the 6.6-cm M-L shaft length. Error bars represent $\pm 1 S E M$.

lines of the figures were $1 \mathrm{~mm}$ thick and drawn with black ink on a white surface. The shaft of each figure was $6.6 \mathrm{~cm}$ long and subtended a visual angle of $18.2^{\circ}$. The tails of the two critical figures were $2.3 \mathrm{~cm}$ long, and the total angle separating the tails was $60^{\circ}$. The tails of the control figure were $2 \mathrm{~cm}$ long.

\section{Results}

It might be expected that trial-to-trial variability in pointing at the shaft endpoints would exceed trial-to-trial variability in estimating visual length, potentially hindering comparison of the two responses. The average withinparticipants standard deviations for the responses, collapsed across the two endpoints for the pointing measures and the three tail directions for the length estimates, gave means of .48 and $.41 \mathrm{~cm}$ for pointing and length estimates, respectively. Even when using a 1-tailed $t$ test, this difference was only marginally statistically significant $(t=1.72, p=.06)$.

To facilitate the comparison of perceived M-L shaft length and egocentric localization of the shaft endpoints, pointing accuracy measures were transformed into "derived lengths" by calculating the difference between the participant's average responses for each of the two endpoints of a given M-L shaft. The results for the visual and pointing measures of length are presented in Figure 2. A tail direction (in/up/out) $\times$ measure (visual/pointing) analysis of variance (ANOVA) produced a main effect for tail direction $[F(2,22)=23.22, p<.001]$ and a statistically significant interaction $[F(2,22)=16.50, p<.001]$. It can be seen from Figure 2 that the interaction is indicative of a systematic increase in perceived length from tails-in, to control, to tails-out figures, but only a small difference in pointing (derived) length. Post hoc contrasts indicated that each of the figures differed statistically $(p<.05)$ from each other on the length measure, but not on the pointing measure. Furthermore, none of the pointing effects differed statistically from zero. In short, despite the overestimation of tails-out shaft length and underestimation of tails-in shaft length, there was no evidence of egocentric mislocalization of the shaft endpoints according to the pointing measures. Nor was there a statistically significant correlation between the length illusion and the derived extent illusion $(r=-.02, p>.05$, and $r=.23$, $p>.05$ for tails-in and tails-out figures, respectively).

To compare the magnitude of the tails-in and tailsout illusions, each observer's M-L length illusion was transformed into a percentage of the perceived length of the control (tails-up) figure according to the following formulas:

$$
\begin{aligned}
& \% \text { tails-in illusion }= \\
& \quad[(\text { tails-up minus tails-in }) / \text { tails-up }] \times 100 \\
& \% \text { tails-out illusion }= \\
& \quad \text { [(tails-out minus tails-up }) / \text { tails-up }] \times 100 .
\end{aligned}
$$

Thus, the numerators of the formulas were so arranged that a positive value signified a percentage difference in the direction predicted by the illusion (i.e., underestimation for the tails-in figure and overestimation for the tailsout figure).

The differences in settings between each of the two critical figures and the control figure, averaged over participants, are presented in Table 1 . The total length illu-

\begin{tabular}{|c|c|c|c|c|c|c|}
\hline \multirow[b]{2}{*}{ Measure } & \multicolumn{4}{|c|}{ Chevron Tail Direction } & \multirow[b]{2}{*}{ Total } & \multirow[b]{2}{*}{$\%$} \\
\hline & In & $\%$ & Out & $\%$ & & \\
\hline Length & $0.52 *$ & 7.5 & $1.12 *$ & 17.0 & $1.64 *$ & 24.5 \\
\hline Pointing & 0.1 & 0.6 & 0.2 & 2.8 & 0.2 & 3.4 \\
\hline
\end{tabular}
sion of $24.5 \%$ is comparable to that of previous studies

Table 1

Experiment 1: Length and Pointing Illusions (in Centimeters)

Note-Percentages are for tails-in or tails-out figure estimate relative to the control figure estimate. ${ }^{*} p<.001$. 
(e.g., Coren \& Girgus, 1978), as are the separate effects for the tails-in $(7.5 \%)$ and tails-out $(17.0 \%)$ figures. In contrast to the visually perceived lengths, the derived pointing lengths revealed average percentage "illusions" of only $0.6 \%$ and $2.8 \%$ for tails-in and tails-out figures, respectively, neither of which was statistically different from zero.

\section{Discussion}

The results of this experiment replicated those of Mack et al. (1985, Experiment 1) and are thus congruent with the hypothesis that the M-L length illusion does not affect egocentric localization. Besides supporting the noncommensurability of length perception and egocentric localization with respect to this stimulus configuration, the results contradict any accounts of the M-L illusion in which mislocalization of the shaft endpoints is supposed to be involved (e.g., Coren \& Girgus, 1978; Ginsburg, 1984; Judd, 1905; Pressey, 1971).

One potential problem with this experiment is the likelihood that the participants' oculomotor behavior differed between the visual and pointing measures. Thus, when pointing at a shaft endpoint, the participants' natural tendency was to fixate that endpoint and ignore the rest of the figure, whereas with the visual measures they most likely scanned the full length of the shaft. It is possible that fixating a small segment of the M-L figure temporarily attenuates or even abolishes the length illusion, due to narrowed attention (e.g., Benussi, 1904). If so, accuracy in localizing the M-L shaft endpoints would be due to the absence of the length illusion itself, rather than a dissociation between perceived length and location. In order to control for eye fixation, a follow-up study $(N=12)$ was carried out. In this study, exactly the same procedure was used as in Experiment 1, except that the participants were required to fixate one endpoint on half of the visual length measures and the other endpoint on the other half. The results were virtually identical to those of Experiment 1: a tails-in illusion of $8.19 \%$ and a tails-out illusion of $17.23 \%$ (total $=25.42 \%$ ), but only small, nonstatistically significant pointing errors.

Thus, the present results and those of Mack et al. (1985) support the claim that visual localization and visual length perception are dissociated from one another with respect to the M-L illusion. However, an alternative possibility is suggested by the so-called perspective theory of visual illusions proposed by Gregory (1963). According to this notion, the M-L length illusion occurs because the chevrons provide 2-D cues for depth that make the shaft of the tailsout figure appear farther away than the shaft of the tails-in figure. Because the retinal images produced by the two shafts are equal, the one seen to be farther away appears larger because of size constancy scaling. If this theory is correct, the absence of pointing errors can be explained without resorting to dissociation between perceived length and perceived location. This interpretation is illustrated in Figure 3. Lines A and B subtend the same visual angle, but Line A is perceived as farther away due to the perspective cues provided by the chevrons. When projected on a frontal plane ( $\mathrm{C}$ in the figure), the line ends would project to the same location, and thus pointing responses to them would be the same.

Evidence contrary to the perspective theory of the M-L illusion and its account of the absence of localization errors comes from examining a variant of the M-L figure that was described by Judd (1899) and is therefore sometimes referred to as the Judd illusion (e.g., Post $\&$ Welch, 1996). As seen in Figures 4A and 4B, this is simply an M-L figure with one of its chevrons rotated to point in the same direction as the other. According to the perspective theory, the fins of this figure, like those of the traditional M-L figure, should cause the shafts to appear displaced away from or toward the observer. That is, because this configuration is equivalent to Mach's (1959) reversible "open book" figure, the spine (shaft) should sometimes appear closer and sometimes farther away. According to the perspective theory, this should produce a corresponding fluctuation in perceived size (see Holding, 1970). However, this figure does not produce an obvious length illusion. And, more importantly for the present investigation, it has a dramatic effect on perceived location (see Figure 4). Thus, the apparent midpoint of the horizontal line is displaced in the direction of the tails-out chevron (Judd, 1899, p. 244), as the reader can attest by attempting to bisect the shafts in Figures 4A and 4B. Even more striking is the mislocalization of the entire figure, an effect readily seen by comparing the apparent locations of the shafts of Figures 4A and 4B. Both Coren (1986, Experiment 5) and Post and Welch (1996) have shown that this mislocalization is egocentric rather than

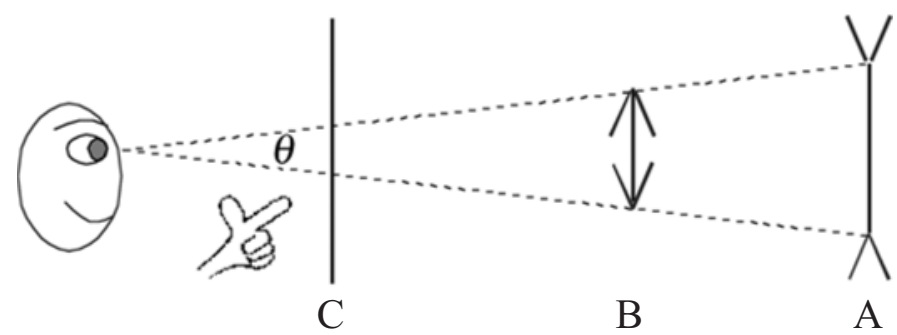

Figure 3. A schematic showing how the perspective theory of the Müller-Lyer illusion can explain the apparent dissociation of perceived location and perceived length. 
A

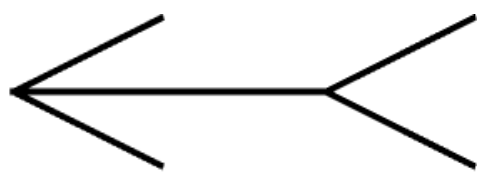

B

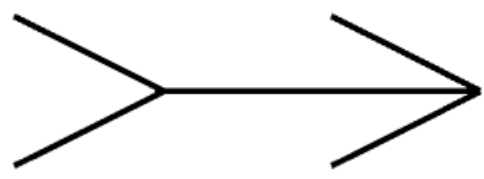

C

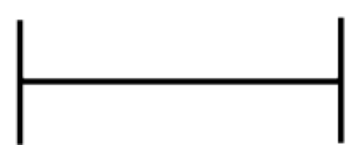

Figure 4. (A) Tails-right, (B) tails-left Judd figures, and (C) tails-up (control).

exocentric, as demonstrated by the substantial errors observers make when attempting to point open loop at the shaft endpoints and apparent bisectors.

The dramatic difference between the perceptual effects of the M-L illusion and those of the Judd illusion clearly hinges on whether the two chevrons are oriented in the same or opposite directions. Thus, in some unknown fashion, the presence of oppositely oriented chevrons appears to suppress or mask the egocentric mislocalization that would otherwise occur. It would thus seem reasonable to predict that if one of these chevrons were removed, the result would be a figure that causes misperception of both length and localization. Experiment 2 tested this prediction with respect to localization.

\section{EXPERIMENT 2}

This experiment was similar to the preceding research, except that the stimuli entailed only one chevron and only endpoint localization was tested. The magnitude of the length illusion for this version of the figure was investigated in Experiment 3 (see also Greene \& Nelson, 1997, and Nelson \& Greene, 1998).

\section{Method}

Participants. Eleven participants ( 6 males and 5 females) with an average age of about 23 years were obtained from the same population as in Experiment 1.

Procedure. The participants fixated an X located on their midline directly in front of the "bare" end of the initially shielded M-L shaft. After a 6-sec fixation period, the poster board sheet on which the $\mathrm{X}$ was attached was raised to reveal the figure. While still fixating where the $X$ had been, the participants pointed open loop at the endpoint of the chevron end of the shaft that was either to the left or right of fixation.

Stimuli. The one-chevron M-L figures are depicted in Figure 5. Their shafts were $3.25 \mathrm{~cm}$ long, exactly one-half those of the twochevron figures of Experiment 1, whereas chevron tail length and separation angle were unchanged. On half of the trials, the chevron was on the left side (as shown in Figure 5), and on the remaining trials it was on the right side.

\section{Results}

In Experiment 1 (and the follow-up study), the participants' average pointing error for one endpoint was subtracted from the average error for the other endpoint to produce a measure of the derived apparent (pointing) length of the shaft. However, it was not possible to use the analogous procedure in Experiment 2 because the participants were required to point only at the shaft endpoint where the chevron was located. Because we cannot know whether the endpoint of the other ("bare") end of the shaft was correctly localized, it would be risky to assume that the (halved) difference between the average locations of the chevron is a valid measure of the derived length of the shaft. Consequently, statistical analyses were performed on the differences between pointing accuracy and the true locus of the chevron-endpoint, averaged over the left and right chevron-endpoint loci, with positive differences indicating shifts from that endpoint in the direction of the observer's visual periphery. The difference scores obtained in this manner were 1.13, 1.03, and $1.40 \mathrm{~cm}$ for tails-in, control, and tails-out figures, respectively. Subtracting the response for the control figure from that for the tails-in and tails-out figures provides a measure of the effect of the illusory figures on pointing accuracy. The results were -.10 and $.37 \mathrm{~cm}$ for tails-in and tails-out figures, respectively. According to $t$ tests, the error for the tails-in figure was not statistically different from zero $[t(10)=-1.26, p>.05]$, whereas that for the tailsout figure was highly significant $[t(10)=6.35, p<.001]$.

\section{Discussion}

The results of this experiment confirm the prediction that for M-L figures with only one chevron, the egocentric location of the end of the shaft to which that chevron
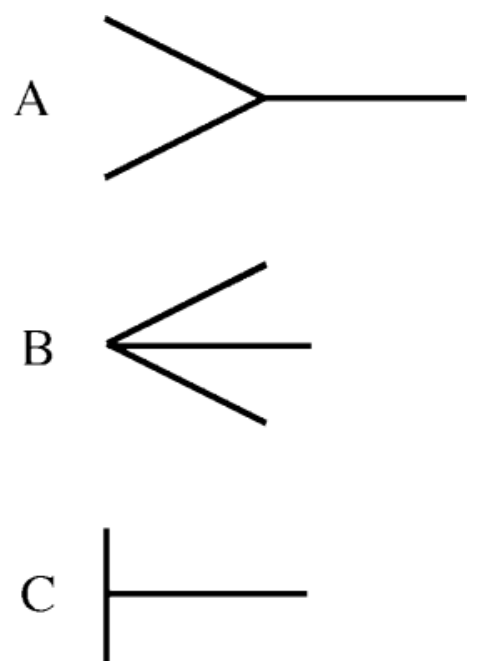

Figure 5. (A) Tails-out, (B) tails-in, and (C) tails-up (control) one-chevron Müller-Lyer figures. 
is attached (and perhaps part of the shaft as well) is displaced in the direction of the chevron's tails. However, this was true only for the tails-out figure. The egocentric mislocalization of the tails-out M-L figure casts a very different light on the absence of such a shift for the traditional two-chevron figure. Specifically, it indicates that, whereas the shaft endpoints of the latter figure are not subject to significant mislocalization, the perceived location of an individual shaft endpoint will be misperceived if there is no oppositely oriented chevron at the other end of the shaft to contradict it. In short, it appears that with the traditional two-chevron tails-out figure, two mislocalization tendencies are occurring but are somehow nullified. Our failure to find evidence for a reliable pointing error for the tails-in figure is perplexing but might indicate merely that this figure is a very poor source of either length or location illusions.

Experiment 3 tested the prediction that one-chevron M-L figures also cause misperceived length. It would seem reasonable to expect this illusion to be less than that found with the traditional two-chevron figure.

\section{EXPERIMENT 3}

\section{Method}

The participants were obtained from the same population as in Experiments 1 and 2. It was necessary to replace 4 of them from the original sample for failing to show a large illusion. The final sample of 10 ( 3 males and 7 females) had an average age of approximately 28.5 years. They were exposed to the same tails-out and tails-up control figures used in the preceding experiment (Figure 5), but not the tails-in figure because of the equivocal results obtained for that stimulus. As in the preceding experiment, both left and right orientations of the two figures were presented, and the participants' fixation point was maintained at the bare end of the shaft, which was located in their midline. The participants pointed open loop at the endpoint on which the single chevron was located. Perceived shaft length was measured by means of the procedure used previously.

\section{Results}

The results for the pointing measures were similar to those of Experiment 2. The control and tails-out errors relative to true endpoint locus (averaged over left and right figure orientations) were .73 and $1.30 \mathrm{~cm}$, respectively, both peripheral to the true locus of the endpoint. As in the preceding experiment, the difference between these two figures $\left(.57 \mathrm{~cm} / 1.56^{\circ}\right.$ of visual angle $)$ provides an estimate of the illusory effect of the figure. According to a $t$ test, this difference was statistically significant $[t(9)=5.50, p<.001]$, indicative of a localization error in the direction predicted for the figure.

The length illusion with the one-chevron figure was robust. The control and tails-out shaft length estimates (averaged over left and right figure orientations) were 3.80 and $4.73 \mathrm{~cm}$, respectively. The difference of $.93 \mathrm{~cm}$ $\left(2.55^{\circ}\right.$ of visual angle $)$, which was statistically significant $[t(9)=5.38, p<.001]$, confirms the predicted overestimation of the tails-out figure. The percentage illusion, according to the usual formula, was a surpris- ingly large $24.5 \%$. The correlation between the two measures was .53 which, with only 10 participants, was not statistically significant $(p>.05)$.

\section{Discussion}

The results of this study revealed that an M-L figure with one (tails-out) chevron continues to produce an illusion of visual length, replicating the results of Greene and Nelson (1997), and Nelson and Greene (1998). The fact that this illusion, when taken as a percentage of the control figure estimate, was (nonsignificantly) greater than that found with the two-chevron figures of Experiment $1(24.5 \%$ vs. $17.2 \%)$ may be misleading, however, because it is based on a shaft length half that of the twochevron figures. Thus, because the effect of a chevron on perceived shaft length declines with distance along the shaft (Post, Welch, \& Caufield, 1998), it is to be expected that when chevron characteristics are held constant, the shorter the shaft, the greater the illusion, when calculated as a percent of the perceived length of the control shaft. Support for this argument is seen in a study by Redding and Hawley (1993), who also compared oneand two-chevron configurations. Unlike the present study, they equated shaft length for the two kinds of figure and found that their one-chevron figures produced only half as much percentage illusion as their corresponding twochevron figures. Nevertheless, the one-chevron length illusion of $2.55^{\circ}$ of visual angle in Experiment 3 was a full $82 \%$ of the two-chevron illusion of $3.12^{\circ}$ error found in Experiment 1.

As in Experiment 2, the tails-out figure produced a statistically significant pointing error. Thus, we may conclude from the results of both experiments that egocentric location is significantly influenced by an $\mathrm{M}-\mathrm{L}$ figure if that figure is unfettered by an oppositely oriented chevron at the other end of the shaft. In short, under these circumstances, perceived location and perceived extent are no longer completely dissociated, as they appear to be with two-chevron figures.

It is interesting that the correlation coefficient for perceived length and pointing error was higher in this experiment $(r=.53)$ than for the tails-out figure in Experiment $1(r=.22)$, a difference one might expect if the two perceptual capacities were more closely associated for one- than for two-chevron figures. However, this difference was not statistically significant $(Z=.72, p>.05)$.

\section{EXPERIMENT 4}

Although the preceding experiment demonstrated that the chevron end of the shaft was mislocalized, it did not determine whether the entire figure shifted to any extent. To answer that question, the final experiment was carried out, in which participants pointed at both the chevron and bare ends of the shaft. It seemed reasonable to predict that any shift obtained for the latter would be less than for the former due to the presumed decline of the chevron's effect 
as a function of distance along the shaft, as mentioned above (Post et al., 1998).

\section{Method}

Apparatus. The apparatus used for this experiment (Figure 6) differed substantially from that used in the preceding experiments. The participant sat with head pressed against a pair of goggles (not shown in the figure). He/she viewed computer-generated stimulus figures projected by an overhead computer monitor onto an angled mirror which, in turn, caused them to appear located on the surface of a digitizing tablet (touch pad) located $53.3 \mathrm{~cm}$ from his/her eyes. The mirror blocked the participant's view of the hand during the pointing responses. Pointing accuracy in the left-right dimension was measured in terms of touch pad pixels and recorded by the computer. The resolution of the touch pad was 23.6 pixels per $\mathrm{cm}$ $(<.05 \mathrm{~cm})$.

Stimuli and Procedure. The stimuli consisted of the same control and tails-out one-chevron figures used in Experiments 2 and 3 (Figure 5). The visual angle subtended by the shaft of a given figure as viewed at the apparent distance of the touch pad was $10.06^{\circ}$. In blocks of 24 trials, the participants pointed at (1) the chevron end of the control figure, (2) the bare end of the control figure, (3) the chevron end of the tails-out figure, and (4) the bare end of the tailsout figure. For half of the participants, the chevron was on the left side for the first four trial blocks and on the right side for the next four blocks. For the remaining participants, the order of chevron location was reversed.

On each trial, a small luminous cross appeared on the otherwise dark screen, and the participant fixated it. After $1 \mathrm{sec}$, the cross was replaced by an M-L figure. The participant was instructed and trained to continue fixating the cross's previous location (always at the midpoint of the M-L shaft) while reaching out and contacting the touch pad at the perceived location of the chevron or bare end of the shaft. Contact with the touch pad was made by means of a stylus that the participant held in the preferred hand (all participants were righthanded). The participants were informed that the location of the figure in the lateral dimension would be randomly shifted from trial to trial. These locations were straight ahead, 20 pixels $(.85 \mathrm{~cm})$ to the left of straight ahead and 20 pixels to the right of straight ahead. This procedure was designed to minimize the possibility that participants would make a stereotyped pointing response to the same location on every trial.

Eight right-handed participants ( 7 males, 1 female) from the same population as in the preceding experiments were tested in this experiment. The average age was approximately 36 years.

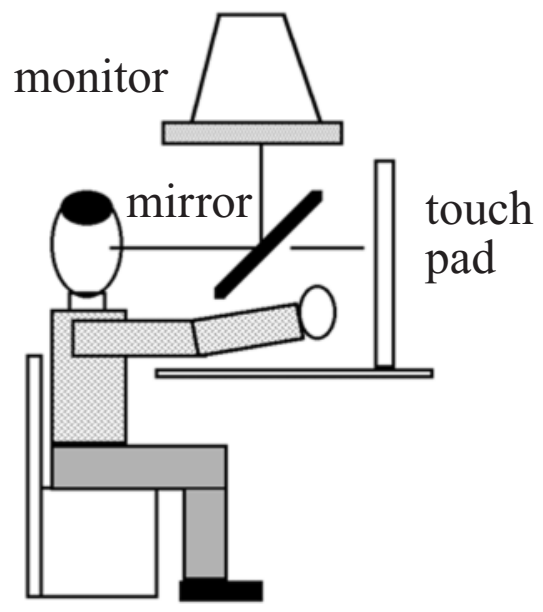

Figure 6. Apparatus for Experiment 4.

\section{Results}

As there was no difference in pointing accuracy for leftoriented and right-oriented figures, these data were combined. The mean pointing errors for bare and chevron endpoints were 14.5 and 21.4 pixels $\left(0.82^{\circ}\right.$ and $1.21^{\circ}$ of visual angle), respectively, a difference that was not statistically significant $[t(7)=.74, p=.48]$. However, the means for both bare and chevron endpoints differed significantly from zero $[t(7)=2.51, p=.04$, and $t(7)=4.89, p<$ .001 , respectively]. An ANOVA on order of figure orientation (left-right/right-left) and shaft endpoint (bare/ chevron) revealed no main effects or interaction $[F(1,6)=$ $1.72, p>.05 ; F(1,6)=0.49, p>.05$; and $F(1,6)=0.29$, $p>.05$, respectively].

\section{Discussion}

The finding that the participants erred in their pointing in the direction of the M-L chevron's tails for both bare and chevron shaft endpoints indicates that the entire figure was at least partially mislocalized. However, although not supported by statistical analysis, there is a suggestion that this error was less for the non-chevron (bare) endpoint. Indeed, a moment's reflection will indicate that with a long enough shaft, no pointing error at all should be observed for the bare end, due to the declining influence of the chevron with increasing shaft distance (Post et al., 1998). To use an extreme example, if the shaft were a meter long, it is highly unlikely that a chevron at one end of the shaft would have any effect at all on pointing to the other end.

\section{GENERAL DISCUSSION}

The present research has shown that the length illusion of the traditional (two-chevron) M-L figure is not accompanied by the errors in egocentric location predicted by strict Euclidean geometry. On the other hand, removing one of the two chevrons caused a "reassociation" in which both length and localization are subject to the illusion. Table 2 summarizes the difference between the effects of one- and two-chevron M-L figures. Only the results for the tails-out figures are presented in this table because this configuration produced the larger, more reliable illusion and was the only one of the two critical M-L figures used in all of the experiments. The length illusion is presented in terms of the degrees of visual angle by which the shaft of the tails-out figure was overestimated relative to the shaft of the control figure. Pointing accuracy was calculated in terms of error relative to the true shaft endpoint loci of the tails-out figure, subtracted from the corresponding error for the control figure and then converted to degrees of visual angle. It can be seen from this table that (1) there is a substantial length illusion for the one-chevron figure ( $82 \%$ of that for the two-chevron figure in Experiment 1) and (2) only with the one-chevron figure is there a reliable illusion-induced shift in egocentric localization. This pattern of results indicates that, for the traditional figure, the visual length illusion su- 
Table 2

Length Illusion and Shaft Endpoint-Pointing Error (in Degrees of Visual Angle) for Tails-Out Müller-Lyer Figures with One Versus Two Chevrons

\begin{tabular}{|c|c|c|c|c|}
\hline & $\begin{array}{l}\text { Length } \\
\text { Illusion }\end{array}$ & $p$ & $\begin{array}{l}\text { Pointing } \\
\text { Accuracy }\end{array}$ & $p$ \\
\hline \multicolumn{5}{|c|}{ Two Chevrons } \\
\hline Experiment 1 & 3.12 & $<.001$ & 0.52 & n.s. \\
\hline Follow-up experiment & 3.18 & $<.001$ & 0.41 & n.s. \\
\hline \multicolumn{5}{|c|}{ One Chevron } \\
\hline Experiment 2 & \multicolumn{2}{|c|}{ - } & 1.00 & $<.001$ \\
\hline Experiment 3 & 2.55 & $<.001$ & 1.56 & $<.001$ \\
\hline Experiment 4 & \multirow{2}{*}{\multicolumn{2}{|c|}{-}} & & \\
\hline (bare end) & & & 0.82 & .04 \\
\hline Experiment 4 & \multirow{2}{*}{\multicolumn{2}{|c|}{ - }} & & \\
\hline (chevron end) & & & 1.21 & .001 \\
\hline
\end{tabular}

persedes the mislocalizing effects, whereas when only one chevron is present, both effects occur.

The egocentric mislocalization of one-chevron M-L figures that we have demonstrated is congruent with other observations. For example, when Benussi (1912) sequentially presented the stimuli depicted in Figure 7A, observers reported that the intersection point, which was in the same location on all three figures, appeared to move left and right. Such illusory motion is what one might expect from the present finding that the apparent locus of a single chevron is mislocalized. Figure 7B depicts the bisector illusion in which the intersection, located at the true midpoint of the horizontal line, is perceived to be to the right of the midpoint, another observation that is congruent with the present results for one-chevron figures.

From the present results and those of previous studies, we may draw the following tentative picture of the role of chevrons in the perception of egocentric location and length. With respect to localization, it can be concluded that a single chevron is mislocalized in the direction of its fins (Experiments 2, 3, and 4), pulling an attached shaft with it, as demonstrated by pointing at the bare end of that shaft (Experiment 4). This effect almost certainly declines with distance from the chevron. Thus, with very short shafts, the entire one-chevron figure should appear shifted in the direction of the fins, whereas with progressively longer shafts, pointing at the bare end will err less and less until it is finally completely accurate. The chevron end, however, will continue to be mislocalized in the direction of the tails. When a relatively short shaft contains an oppositely oriented chevron at each end, the mislocalizing effect of one chevron inhibits or suppresses the mislocalizing effect of the other, resulting in little or no openloop pointing error and thus an apparent severe violation of Euclidean geometry (Experiment 1). Once again, however, this effect will be most pronounced with relatively short figures and progressively less so as shaft length increases (Post et al., 1998) until, in the extreme case, it will disappear altogether, leading to large pointing errors.

With respect to perceived length, we would argue that a single chevron expands space on the side opposite the direction of its fins (Experiment 3), although this effect declines with distance along the shaft (Post et al., 1998). An oppositely oriented chevron on the other end of the shaft also stretches space on the opposite side of its fin's direction. Thus, the two effects combine to produce the well-known M-L length illusion. However, because this effect declines with distance along the shaft, longer and longer shafts will be subject to less and less overestimation (at least as a percentage of the control shaft length estimate). Figure 8 depicts the idealized proposed functional relationship between shaft length and the effects of two opposing chevrons on errors in perceived location and length. It can be seen from the figure that at some point on the continuum of possible shaft lengths (length 6 in the figure), localization errors should equal the length estimate errors that would be expected if Euclidean geometry prevails.

To conclude, the present evidence that the traditional M-L figure does not involve egocentric localization errors casts doubt on any explanation of the M-L illusion based solely on mislocalization of shaft endpoints. More important, however, are the implications of this observation for our understanding of normal perception, which, after all, is the ultimate goal of research on visual illu-
A

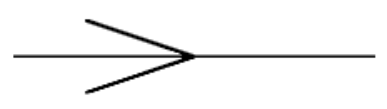

\begin{tabular}{l|l} 
& \\
\hline$\cdot 3 \cdot 1$ & \\
\hline &
\end{tabular}

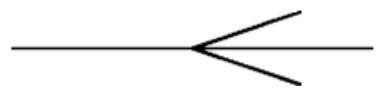

B

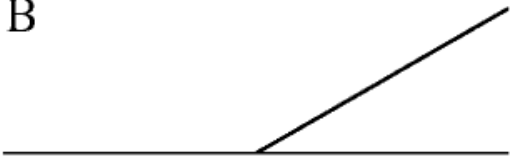

Figure 7. (A) Benussi figures and (B) bisector illusion. 


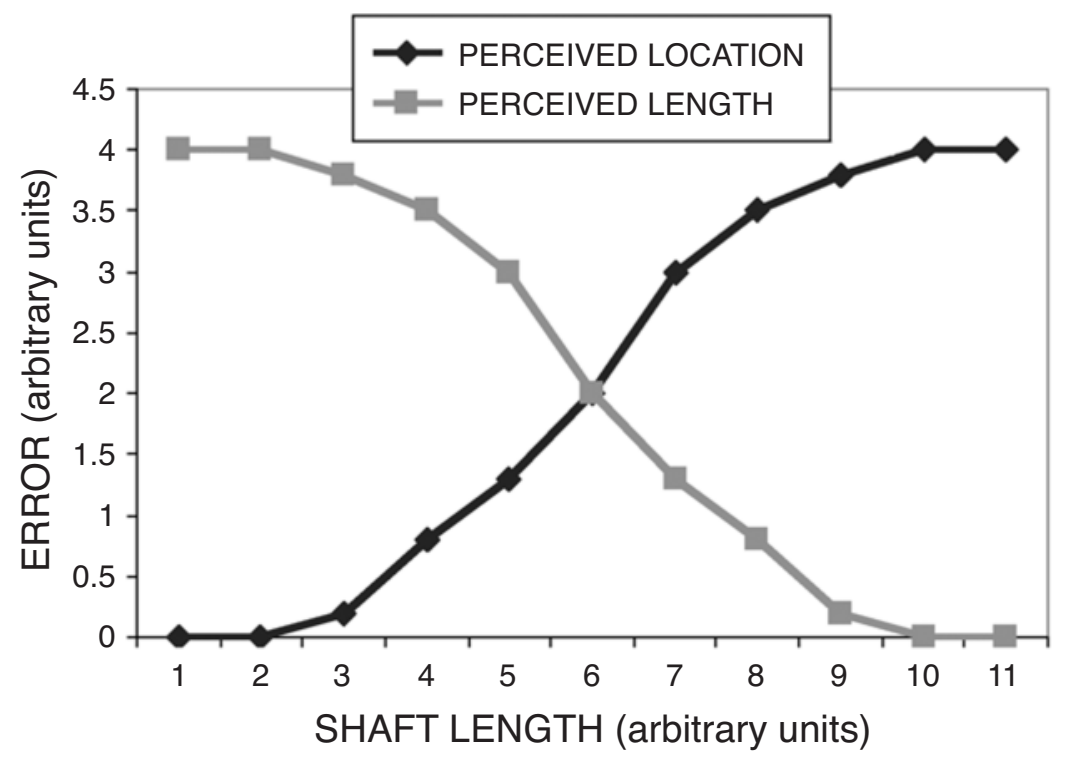

Figure 8. Proposed (idealized) relationship between shaft length of a two-chevron $M-L$ figure and errors of perceived location and length.

sions (e.g., Gregory, 1963). Specifically, the finding suggests that the everyday perceptions of length and location are based on separable mechanisms and neurophysiological substrates. (Exactly what these mechanisms might be is a subject worthy of future study.) Our research has suggested further that this separability with respect to the traditional M-L figure is not due to a passive mechanism in which the figure influences perceived length but not perceived location. If this were the case, removing one of the chevrons would have no effect on egocentric localization of the chevron end of the figure. On the contrary, we have shown that one-chevron M-L figures are substantially mislocalized, implying an underlying process by which the effect of a chevron at one shaft endpoint of the traditional figure actively inhibits the effect of the opposing chevron (except perhaps for very long figures; see Figure 8). Thus, with the seemingly minor alteration of removing one chevron, Euclidean geometry is reinstated in that the resulting figure now produces both length and localization errors.

\section{REFERENCES}

Abrams, R. A., \& Landgraf, J. Z. (1990). Differential use of distance and location information for spatial localization. Perception \& Psychophysics, 47, 349-359.

Benussi, V. (1904). Zur Psychologie des Gestalterfassens (die MüllerLyerische Figur). In A. Meinong (Ed.), Untersuchungen zur Gegenstands Theorie und Psychologie. Leipzig: Johann Ambrosious Burth.

BENussi, V. (1912). Stroboskopische Scheinbewegungen und geometrischoptische Gestalttauschungen. Archiv für die Gesamte Psychologie, 24, 31-62.

CoRen, S. (1986). An efferent component in the visual perception of direction and extent. Psychological Review, 93, 391-410.

Coren, S., \& Girgus, J. S. (1978). Seeing is deceiving: The psychology of visual illusions. Hillsdale, NJ: Erlbaum.
Gentilucci, M., Chieffi, S., Daprati, E., Saetti, M. C., \& Toni, I. (1996). Visual illusion and action. Neuropsychologia, 34, 369-376.

Gillam, B., \& Chambers, D. (1985). Size and position are incongruous: Measurements on the Müller-Lyer figure. Perception \& Psychophysics, 37, 549-556.

GINSBURG, A. P. (1984). Visual form perception based on biological filtering. In L. Spillmann \& B. R. Wooten (Eds.), Sensory experience, adaptation, and perception: Festschrift for Ivo Kohler (pp. 53-72). Hillsdale, NJ: Erlbaum.

Greene, E., \& Nelson, B. (1997). Evaluating Müller-Lyer effects using single fin-set configurations. Perception \& Psychophysics, 59, 293312 .

GREGORY, R. L. (1963). Distortion of visual space as inappropriate constancy scaling. Nature, 199, 678-680.

Holding, H. H. (1970). A line illusion with irrelevant depth cues. American Journal of Psychology, 83, 280-281.

JuDD, C. H. (1899). A study of geometric illusions. Psychological Review, 6, 241-261.

JudD, C. H. (1905). The Müller-Lyer illusion. Psychological Review Monograph Supplement, 7(Whole No. 29), 55-82.

MACH, E. (1959). The analysis of sensations, and the relation of the physical to the psychical (C. M. Williams, Trans., \& S. Waterlow, Ed.). New York: Dover.

Mack, A., Heuer, F., Villardi, K., \& Chambers, D. (1985). The dissociation of position and extent in Müller-Lyer figures. Perception \& Psychophysics, 37, 335-344.

Morgan, M. J., Hole, G. J., \& Glennerster, A. (1990). Biases and sensitivities in geometrical illusions. Vision Research, 30, 1793-1810.

Nelson, B., \& Greene, E. (1998). Similar Müller-Lyer effects from operant and comparison response modes. Perceptual \& Motor Skills, 86, 499-511.

Post, R. B., \& Welch, R. B. (1996). Is there dissociation of perceptual and motor responses to figural illusions? Perception, 25, 569-581.

Post, R. B., Welch, R. B., \& Caufield, K. (1998). Relative spatial expansion and contraction within the Müller-Lyer and Judd illusions. Perception, 27, 827-838.

PRESSEY, A. W. (1971). An extension of assimilation theory to illusions of size, area, and direction. Perception \& Psychophysics, 9, 172-176.

Redding, G. M., \& Hawley, E. (1993). Length illusion in fractional Müller-Lyer stimuli: An object-perception approach. Perception, 22, 819-828. 
ShePard, R. N. (1981). Psychological complementarity. In M. Kubovy \& J. R. Pomerantz (Eds.), Perceptual organization (chapter 10). Hillsdale, NJ: Erlbaum.

WonG, E., \& MACK, A. (1981). Saccadic programming and perceived location. Acta Psychologica, 48, 123-131.

\section{NOTE}

1. It could be argued that because the visual extent measures were made on the basis of the observer's (very short-term) memory of the figure, these responses might differ from those obtained with a currently viewed figure. However, preliminary data using this procedure revealed an M-L length illusion for each of the two critical M-L figures comparable to that commonly reported in the literature (e.g., Coren \& Girgus, 1978).

(Manuscript received January 25, 2000; revision accepted for publication November 24, 2003.) 